\title{
Effect of Heteroatom Insertion at the side-chain of 5-alkyl-1H-tetrazoles on their Properties as Catalysts for Ester Hydrolysis at Neutral pH
}

Santanu Bhattacharya ${ }^{*}$ and Vemula Praveen Kumar

Table of Contents:

Scheme S1

Synthesis

TableS1

Table S2
$\mathrm{S} 2$

S3-S5

S6

S7

Cartesian coordinates of full-optimized geometries S8-S10 


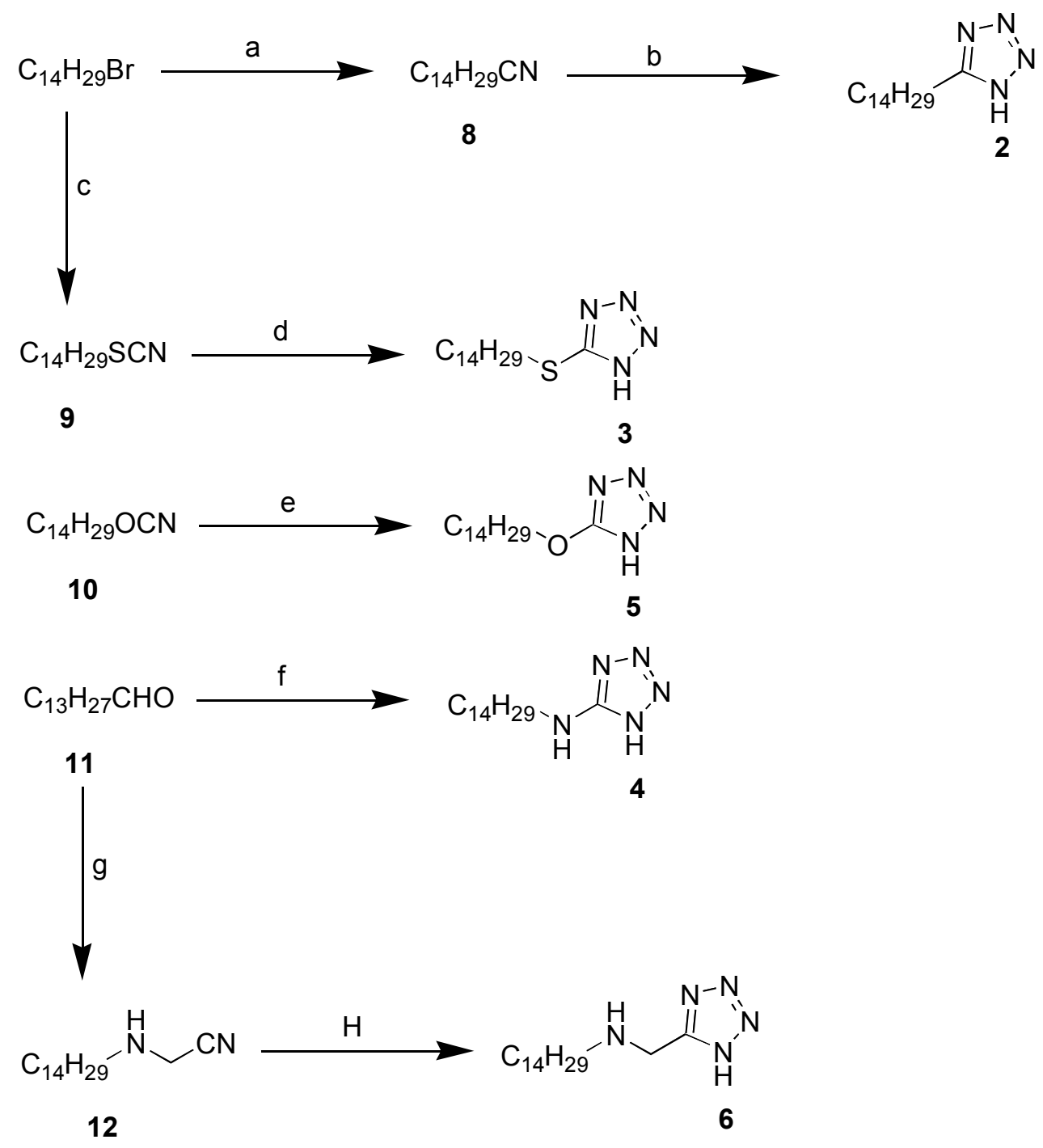

${ }^{\mathrm{a}}$ Reagents, conditions and yields: (a) $\mathrm{NaCN}$, EtOH/ $\mathrm{H}_{2} \mathrm{O}$, reflux, $24 \mathrm{~h}, 91 \%$; (b) $\mathrm{NaN}_{3}, \mathrm{ZnBr}_{2}, \mathrm{H}_{2} \mathrm{O}$, $i$ $\mathrm{PrOH}, 170{ }^{\circ} \mathrm{C}, 36$ h, 78\%; (c) KSCN, EtOH/H ${ }_{2} \mathrm{O}$, reflux, 28 h, 94\%; (d) $\mathrm{NaN}_{3}, \mathrm{ZnBr}_{2}, \mathrm{H}_{2} \mathrm{O}, \mathrm{EtOH}, 110$ ${ }^{\circ} \mathrm{C}, 36 \mathrm{~h}, 81 \%$; (e) $\mathrm{NaN}_{3}, \mathrm{ZnBr}_{2}, \mathrm{H}_{2} \mathrm{O}, \mathrm{EtOH}, 110{ }^{\circ} \mathrm{C}, 18 \mathrm{~h}, 63 \%$; (f) i) 5-Aminotetrazole hydrochloride, $\mathrm{MeOH} / \mathrm{CHCl}_{3}, \mathrm{NEt}_{3}, 50{ }^{\circ} \mathrm{C}, 6 \mathrm{~h}$; ii) $\mathrm{NaBH}_{4}$, r.t., $12 \mathrm{~h}, 58 \%$; (g) Aminoacetonitrile hydrochloride, $\mathrm{MeOH} / \mathrm{CHCl}_{3}, \mathrm{NEt}_{3}, 50{ }^{\circ} \mathrm{C}, 6$ h; (ii) $\mathrm{NaBH}_{4}$, r.t., 12 h, 46\%; (h) $\mathrm{NaN}_{3}, \mathrm{ZnBr}_{2}, \mathrm{EtOH} / \mathrm{H}_{2} \mathrm{O}, 140{ }^{\circ} \mathrm{C}, 24$ h, $62 \%$. 


\section{Synthesis.}

General Procedure for the Conversion of Nitriles into Tetrazoles. To a round-bottom flask (100 $\mathrm{mL})$ containing water $(20 \mathrm{~mL})$ was added the nitrile $(10 \mathrm{mmol})$, sodium azide $(0.71 \mathrm{~g}, 11 \mathrm{mmol}), \mathrm{ZnBr}_{2}$ $(8.25 \mathrm{~g}, 10 \mathrm{mmol})$, and $5 \%(\mathrm{v} / \mathrm{v})$ co-solvent (either $i-\mathrm{PrOH}$ or EtOH). The reaction mixture was refluxed for $24-48 \mathrm{~h}$ with vigorous stirring. Then the mixture was cooled to room temperature, the $\mathrm{pH}$ was adjusted to 1.0 with concentrated $\mathrm{HCl}(3 \mathrm{~N}, 15 \mathrm{~mL})$, and ethyl acetate $(50 \mathrm{~mL})$ was added, and vigorous stirring was continued until no solid was present and the aqueous layer had a $\mathrm{pH}$ of 1.0. The organic layer was isolated and the aqueous layer extracted with ethyl acetate $(2 \times 75 \mathrm{~mL})$. The combined organic layers were evaporated, $100 \mathrm{~mL}$ of $0.25 \mathrm{~N} \mathrm{NaOH}$ was added, and the mixture was stirred for $30 \mathrm{~min}$, until the original precipitate was dissolved and a suspension of zinc hydroxide was formed. The suspension was filtered, and the solid washed with $10 \mathrm{~mL}$ of $1 \mathrm{~N} \mathrm{NaOH}$. To the filtrate 20 $\mathrm{mL}$ of $3 \mathrm{~N} \mathrm{HCl}$ was added with vigorous stirring. This caused the precipitation of tetrazole, which was filtered and washed with $3 \mathrm{~N} \mathrm{HCl}(2 \times 10 \mathrm{~mL})$ and dried to furnish the pure tetrazole as solid.

1-Cyanotetradecane (8). In a round-bottom flask $(100 \mathrm{~mL}), n$-tetradecyl bromide $(0.83 \mathrm{~g}, 3 \mathrm{mmol})$ was dissolved in $10 \mathrm{~mL}$ of $\mathrm{EtOH}: \mathrm{H}_{2} \mathrm{O}(9: 1)$ mixture. To this $\mathrm{NaCN}(0.44 \mathrm{~g}, 9 \mathrm{mmol})$ was added and the resulting mixture was refluxed for $24 \mathrm{~h}$. The excess ethanol was removed under vacuo, $50 \mathrm{~mL}$ of water was added and the aqueous layer extracted with EtOAc $(3 \times 25 \mathrm{~mL})$. Combined organic layers were passed through anhydrous $\mathrm{Na}_{2} \mathrm{SO}_{4}$ and concentrated to leave a residue which was purified by column chromatography over silica gel using EtOAc/hexane (3:97). The pure product, 8 was obtained ${ }^{1}$ as a viscous liquid (0.61 g, 91\%). IR: (neat) 2926, 2246, $1465 \mathrm{~cm}^{-1} ;{ }^{1} \mathrm{H}-\mathrm{NMR}:\left(\mathrm{CDCl}_{3}, 300 \mathrm{MHz}\right) \delta 2.32(t$, 2H), $1.66(q, 2 \mathrm{H}), 1.25(m, 22 \mathrm{H}), 0.88(t, 3 \mathrm{H}) ; \mathrm{EI}-\mathrm{MS}, \mathrm{m} / \mathrm{z} 224\left(\mathrm{M}^{+}\right)$.

1-Thiocyanato tetradecane (9). Compound 9 was synthesized by using similar procedure, which we used for synthesis of 8 in 94\% yield. IR: (neat) 2924, 2155, $1464 \mathrm{~cm}^{-1} ;{ }^{1} \mathrm{H}-\mathrm{NMR}:\left(\mathrm{CDCl}_{3}, 300 \mathrm{MHz}\right) \delta$ $2.92(t, 2 \mathrm{H}), 1.81(m, 2 \mathrm{H}), 1.24(m, 22 \mathrm{H}), 0.87(t, 3 \mathrm{H}) ; \mathrm{EI}-\mathrm{MS}, \mathrm{m} / \mathrm{z} 256\left(\mathrm{M}^{+}\right)$; Anal. Calcd. for $\mathrm{C}_{15} \mathrm{H}_{29} \mathrm{SN}: \mathrm{C}, 70.55 ; \mathrm{H}, 11.44 ; \mathrm{N}, 5.48$. Found: $\mathrm{C}, 70.51 ; \mathrm{H}, 11.47 ; \mathrm{N}, 5.51$. 
(N-tetradecyl)aminoacetonitrile (12). Compound 12 was synthesized by using a similar procedure as adopted for $\mathbf{4}$ by reacting 1-tetradecanal with aminoacetonitrile hydrochloride. Pure $\mathbf{1 2}$ was obtained in 46\% yield. IR: (neat) 3323, 2917, 2250, $1644 \mathrm{~cm}^{-1} ;{ }^{1} \mathrm{H}-\mathrm{NMR}:\left(\mathrm{CDCl}_{3}, 300 \mathrm{MHz}\right) \delta 5.89$ (br $\left.s, 1 \mathrm{H}\right)$, $4.19(d, 2 \mathrm{H}), 2.22(t, 2 \mathrm{H}), 1.61(m, 2 \mathrm{H}), 1.25(m, 22 \mathrm{H}), 0.88(t, 3 \mathrm{H}) ;$ EI-MS, m/z $252\left(\mathrm{M}^{+}\right)$; Anal. Calcd. for $\mathrm{C}_{16} \mathrm{H}_{32} \mathrm{~N}_{2}$ : C, 76.12; H, 12.78; N, 11.10. Found: C, 76.14; H, 12.74; N, 11.12.

5-(n-Tetradecyl) $\mathbf{1 H}$-tetrazole (2). 2-PrOH (3 mL) was added to the reaction mixture to facilitate solubilization of the nitrile. The reaction mixture was refluxed at $170{ }^{\circ} \mathrm{C}$ for $36 \mathrm{~h}$. The pure product was obtained as white solid in 78\% yield. IR: $(\mathrm{KBr}) 3225,2916,1546,1471,1075 \mathrm{~cm}^{-1}$; ${ }^{1} \mathrm{H}-\mathrm{NMR}$ : $\left(\mathrm{CDCl}_{3^{-}}\right.$ DMSO- $\left.d_{6}, 300 \mathrm{MHz}\right) \delta 3.02(t, 2 \mathrm{H}), 1.83(m, 2 \mathrm{H}), 1.26(m, 22 \mathrm{H}), 0.87(t, 3 \mathrm{H}) ; \mathrm{EI}-\mathrm{MS}, \mathrm{m} / \mathrm{z} 267\left(\mathrm{M}^{+}\right)$; Anal. Calcd. for $\mathrm{C}_{15} \mathrm{H}_{30} \mathrm{~N}_{4}$ : C, 67.62; H, 11.34; N, 21.03. Found: C, 67.69; H, 11.41; N, 20.91.

5-(n-Tetradecylthio) $1 H$-tetrazole (3). Earlier Lieber and Enkoji reported the synthesis of various thiolated derivatives of $1 H$-tetrazoles. ${ }^{2}$ EtOH $(3 \mathrm{~mL})$ was used as co-solvent of the reaction mixture to facilitate solubilization of the nitrile. The reaction mixture was heated at $110{ }^{\circ} \mathrm{C}$ for $36 \mathrm{~h}$. Pure 3 was isolated as a white solid in $81 \%$ yield. IR: $(\mathrm{KBr}) 3463,2919,1485,1064 \mathrm{~cm}^{-1}$; ${ }^{1} \mathrm{H}-\mathrm{NMR}$ : $\left(\mathrm{CDCl}_{3^{-}}\right.$ DMSO- $\left.d_{6}, 300 \mathrm{MHz}\right) \delta 3.11(t, 2 \mathrm{H}), 1.68(m, 2 \mathrm{H}), 1.17(m, 22 \mathrm{H}), 0.79(t, 3 \mathrm{H}) ; \mathrm{EI}-\mathrm{MS}, \mathrm{m} / \mathrm{z} 299\left(\mathrm{M}^{+}\right)$; Anal. Calcd. for $\mathrm{C}_{15} \mathrm{H}_{30} \mathrm{~N}_{4} \mathrm{~S}$ : C, 60.36; H, 10.13; N, 18.77. Found: C, 60.72; H, 10.31; N, 18.59 .

5-(n-Tetradecylamino) $1 H$-tetrazole (4). 1-Tetradecanal (0.64 g, $3 \mathrm{mmol})$ was added in one portion to a warm $\left(50{ }^{\circ} \mathrm{C}\right)$ solution of 5 -aminotetrazole $(0.28 \mathrm{~g}, 3.3 \mathrm{mmol})$ and triethylamine $(0.47 \mathrm{~mL}, 3.3$ mmol) in 2:1 mixture of $\mathrm{MeOH}$ and $\mathrm{CHCl}_{3}$. After $6 \mathrm{~h}$, the reaction mixture was cooled to room temparature, $\mathrm{NaBH}_{4}(0.23 \mathrm{~g}, 6 \mathrm{mmol})$ was added in small portions and allowed to stir at room temparature for $12 \mathrm{~h}$. Acidic water was added and saturated with $\mathrm{NaCl}$, then extracted twice with a mixed solvent system of $\mathrm{MeOH}: \mathrm{CHCl}_{3}$ (5:95). Combined organic layers were concentrated, recrystallized using hot EtOAc/MeOH (85:15). Compound 4 was obtained as a solid in 58\% yield. IR: (KBr) 3248, 2934, 1505, $1071 \mathrm{~cm}^{-1}$; ${ }^{1} \mathrm{H}-\mathrm{NMR}:\left(\mathrm{CDCl}_{3}-\mathrm{DMSO}-d_{6}, 300 \mathrm{MHz}\right) \delta 2.41(m, 2 \mathrm{H}), 1.61(m$, 
2H), $1.20(m, 22 \mathrm{H}), 0.82(t, 3 \mathrm{H})$; EI-MS, m/z $282\left(\mathrm{M}^{+}\right)$; Anal. Calcd. for $\mathrm{C}_{15} \mathrm{H}_{31} \mathrm{~N}_{5}$ : C, 64.01; H, 11.1;

N, 24.88. Found: C, 64.14; H, 11.26; N, 24.69.

5-(n-Tetradecyloxy) $\mathbf{1 H}$-tetrazole (5). EtOH (3 mL) was used as co-solvent of the reaction mixture to facilitate solubilization of the nitrile. The reaction mixture was heated at $110{ }^{\circ} \mathrm{C}$ for $18 \mathrm{~h}$. Compound 5 was obtained as a white solid in $63 \%$ in yield. IR: $(\mathrm{KBr}) 3354,2914,1470,1069 \mathrm{~cm}^{-1}$; ${ }^{1} \mathrm{H}-\mathrm{NMR}$ : $\left(\mathrm{CDCl}_{3}-\mathrm{DMSO}-d_{6}, 300 \mathrm{MHz}\right) \delta 4.01(t, 2 \mathrm{H}), 1.84(m, 2 \mathrm{H}), 1.22(m, 22 \mathrm{H}), 0.84(t, 3 \mathrm{H}) ; \mathrm{EI}-\mathrm{MS}, \mathrm{m} / \mathrm{z} 283$ $\left(\mathrm{M}^{+}\right)$; Anal. Calcd. for $\mathrm{C}_{15} \mathrm{H}_{30} \mathrm{~N}_{4} \mathrm{O}: \mathrm{C}, 63.79 ; \mathrm{H}, 10.71 ; \mathrm{N}, 19.84$. Found: C, 64.01; H, 10.67; N, 20.03. 5-((N-tetradecyl)aminomethyly) $1 H$-tetrazole (6). Compound 6 was synthesized by using a similar procedure used for 3 . The pure 6 was obtained as a white solid in 62\% yield. IR: (KBr) 3341, 2924, 1485, $1067 \mathrm{~cm}^{-1} ;{ }^{1} \mathrm{H}-\mathrm{NMR}:\left(\mathrm{CDCl}_{3}\right.$-DMSO-d $\left.6,300 \mathrm{MHz}\right) \delta 5.81(\mathrm{br} s, 1 \mathrm{H}), 4.21(d, 2 \mathrm{H}), 2.25(t, 2 \mathrm{H})$, $1.57(m, 2 \mathrm{H}), 1.21(m, 22 \mathrm{H}), 0.88(t, 3 \mathrm{H}) ; \mathrm{EI}-\mathrm{MS}, \mathrm{m} / \mathrm{z} 294\left(\mathrm{M}^{+}\right)$; Anal. Calcd. for $\mathrm{C}_{16} \mathrm{H}_{33} \mathrm{~N}_{5}: \mathrm{C}, 65.04 ; \mathrm{H}$, 11.25; N, 23.71. Found: C, 65.09; H, 11.19; N, 23.72.

\section{Reference:}

1. Gagnon, P. E.; Boivin, J. L.; Giquire, J. Can. J. Res. 1950, $28 B, 352$.

2. Lieber, E.; Enkoji, T. J. Org. Chem. 1960, 26, 4472. 
Table S1. Comparison of Crystal structural bond lengths $(\AA)$ and bond angles (degrees) of $1 H$-tetrazole, 1, with optimized, computed bond lengths and bond angles at different level of theory. ${ }^{\text {a }}$

\begin{tabular}{|c|c|c|c|c|c|c|c|}
\hline \multirow[b]{2}{*}{ coordinate } & \multicolumn{2}{|c|}{ RHF } & \multicolumn{2}{|c|}{ MP2 } & \multicolumn{2}{|c|}{ B3LYP } & \\
\hline & $6-31 \mathrm{G}^{*}$ & $6-31+\mathrm{G}^{*}$ & $6-31 \mathrm{G}^{*}$ & $6-31+\mathrm{G}^{*}$ & $6-31 G^{*}$ & $6-31+\mathrm{G}^{*}$ & crystal structure \\
\hline
\end{tabular}

$\begin{array}{llllllll}\mathrm{N}_{1}-\mathrm{C}_{5} & 1.33 & 1.33 & 1.347 & 1.349 & 1.347 & 1.347 & 1.33 \\ \mathrm{C}_{5}-\mathrm{N}_{4} & 1.289 & 1.29 & 1.326 & 1.328 & 1.315 & 1.316 & 1.30 \\ \mathrm{~N}_{1}-\mathrm{N}_{2} & 1.326 & 1.326 & 1.348 & 1.348 & 1.353 & 1.353 & 1.33 \\ \mathrm{~N}_{2}-\mathrm{N}_{3} & 1.252 & 1.252 & 1.322 & 1.324 & 1.292 & 1.291 & 1.30 \\ \mathrm{~N}_{3}-\mathrm{N}_{4} & 1.341 & 1.341 & 1.363 & 1.363 & 1.366 & 1.365 & 1.33 \\ \mathrm{~N}_{1}-\mathrm{H}_{6} & 0.994 & 0.994 & 1.01 & 1.01 & 1.01 & 1.01 & 1.10 \\ \mathrm{~N}_{1}-\mathrm{N}_{2}-\mathrm{N}_{3} & 106.6 & 106.7 & 105.3 & 105.3 & 105.8 & 106.0 & 107.6 \\ \mathrm{~N}_{1}-\mathrm{C}_{5}-\mathrm{N}_{4} & 108.1 & 108.1 & 108.2 & 108.1 & 108.4 & 108.3 & 106.7 \\ \mathrm{C}_{5}-\mathrm{N}_{1}-\mathrm{N}_{2} & 108.0 & 108.0 & 109.5 & 109.5 & 108.6 & 108.6 & 108.5 \\ & & & & & & & \end{array}$

${ }^{\text {a}}$ See reference: El-Azhary, A. A.; Suter, H. U.; Kubelka, J. J. Phys. Chem. A 1998, 102, 620. for crystal structure data. 
Table S2. Comparison of Crystal structural bond lengths $(\AA)$ and bond angles (degrees) of terazolyl anion (conjugate base of $\mathbf{1}$ ) with optimized bond lengths and bond angles at different level of theory. ${ }^{\text {a }}$

\begin{tabular}{|c|c|c|c|c|c|c|c|}
\hline \multirow[b]{2}{*}{ coordinate } & \multicolumn{2}{|c|}{ RHF } & \multicolumn{2}{|c|}{ MP2 } & \multicolumn{2}{|c|}{ B3LYP } & \\
\hline & $6-31 \mathrm{G}^{*}$ & $6-31+\mathrm{G}^{*}$ & $6-31 \mathrm{G}^{*}$ & $6-31+\mathrm{G}^{*}$ & $6-31 G^{*}$ & $6-31+\mathrm{G}^{*}$ & crystal structure \\
\hline
\end{tabular}

$\begin{array}{llllllll}\mathrm{N}_{1}-\mathrm{C}_{5} & 1.316 & 1.317 & 1.345 & 1.348 & 1.339 & 1.340 & 1.329 \\ \mathrm{C}_{5}-\mathrm{N}_{4} & 1.316 & 1.317 & 1.345 & 1.348 & 1.339 & 1.340 & 1.329 \\ \mathrm{~N}_{1}-\mathrm{N}_{2} & 1.322 & 1.321 & 1.360 & 1.361 & 1.353 & 1.352 & 1.348 \\ \mathrm{~N}_{2}-\mathrm{N}_{3} & 1.282 & 1.282 & 1.348 & 1.349 & 1.324 & 1.323 & 1.310 \\ \mathrm{~N}_{3}-\mathrm{N}_{4} & 1.322 & 1.321 & 1.360 & 1.361 & 1.353 & 1.352 & 1.348 \\ \mathrm{~N}_{1}-\mathrm{N}_{2}-\mathrm{N}_{3} & 110.0 & 110.0 & 109.4 & 109.4 & 109.7 & 109.6 & 109.5 \\ \mathrm{~N}_{1}-\mathrm{C}_{5}-\mathrm{N}_{4} & 112.5 & 112.2 & 113.7 & 113.3 & 113.4 & 112.9 & 112.5 \\ \mathrm{C}_{5}-\mathrm{N}_{1}-\mathrm{N}_{2} & 103.7 & 103.9 & 103.6 & 103.9 & 103.5 & 103.8 & 104.3\end{array}$

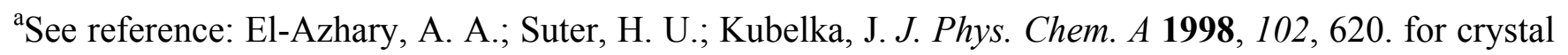
structure data. 
Cartesian coordinates of full-optimized geometries of 2-6 and their conjugate bases at B3LYP/6-31+G*.

\begin{tabular}{rrrr}
\multicolumn{2}{c}{$\mathbf{2}$} & & \\
6 & 2.472034 & 0.173586 & -0.563430 \\
6 & 1.556846 & -0.267430 & 0.595274 \\
1 & 3.522779 & 0.044616 & -0.282272 \\
1 & 2.280579 & -0.426172 & -1.459141 \\
1 & 2.319786 & 1.228203 & -0.821562 \\
6 & 0.102048 & -0.156751 & 0.267788 \\
1 & 1.748196 & -1.315705 & 0.843828 \\
1 & 1.778071 & 0.322499 & 1.494188 \\
7 & -0.629121 & 0.981671 & 0.258240 \\
1 & -0.374364 & 1.933085 & 0.488680 \\
7 & -1.898247 & 0.695327 & -0.118909 \\
7 & -0.702236 & -1.139268 & -0.102137 \\
7 & -1.921912 & -0.578152 & -0.331697
\end{tabular}

\section{Conjugate base of 2}

$\begin{array}{lll}-1.890161 & 0.661579 & -0.228933\end{array}$

$\begin{array}{lll}-1.890571 & -0.660473 & -0.230169\end{array}$

$\begin{array}{lll}-0.660811 & -1.113853 & 0.098445\end{array}$

$\begin{array}{lll}0.064840 & -0.000547 & 0.293469\end{array}$

$\begin{array}{lll}-0.660146 & 1.113574 & 0.100606\end{array}$

$\begin{array}{lll}1.524124 & -0.001335 & 0.641341\end{array}$

$\begin{array}{lll}1.744485 & 0.882807 & 1.255120\end{array}$

$\begin{array}{lll}1.744232 & -0.887913 & 1.251677\end{array}$

$\begin{array}{llll}2.444863 & 0.000982 & -0.594321\end{array}$

$\begin{array}{llll}3.506736 & 0.000341 & -0.304310\end{array}$

$\begin{array}{llll}2.256640 & -0.883936 & -1.214249\end{array}$

$\begin{array}{llll}2.256765 & 0.888314 & -1.210821\end{array}$

3

$\begin{array}{crrr}6 & -2.329385 & 0.879903 & 0.000387 \\ 16 & -1.494970 & -0.748102 & -0.000075 \\ 1 & -3.399710 & 0.658574 & 0.000926 \\ 1 & -2.096482 & 1.454605 & 0.901772 \\ 1 & -2.097432 & 1.454664 & -0.901206 \\ 6 & 0.188493 & -0.263146 & -0.000049 \\ 7 & 0.705366 & 0.987509 & -0.000162 \\ 1 & 0.261289 & 1.894888 & -0.000233 \\ 7 & 1.198792 & -1.118747 & 0.000077 \\ 7 & 2.063570 & 0.896209 & -0.000021 \\ 7 & 2.331873 & -0.364062 & -0.000192\end{array}$

Conjugate base of 3 


$\begin{array}{crrr}6 & 0.004311 & 0.314226 & 0.000000 \\ 7 & -0.029947 & 1.652576 & 0.000000 \\ 7 & -1.355917 & 1.933004 & 0.000000 \\ 7 & -2.051241 & 0.814585 & 0.000000 \\ 7 & -1.210297 & -0.250853 & 0.000000 \\ 16 & 1.541754 & -0.578517 & 0.000000 \\ 6 & 0.908838 & -2.293170 & 0.000000 \\ 1 & 1.785521 & -2.950511 & 0.000000 \\ 1 & 0.299670 & -2.482363 & 0.887547 \\ 1 & 0.299670 & -2.482363 & -0.887547\end{array}$

\begin{tabular}{rrrr}
\multicolumn{4}{c}{4} \\
6 & -2.497847 & -0.367836 & 0.041280 \\
7 & -1.436381 & 0.633495 & -0.084712 \\
1 & -3.454202 & 0.125336 & -0.148417 \\
1 & -2.343958 & -1.145541 & -0.709277 \\
1 & -2.515657 & -0.840880 & 1.031766 \\
6 & -0.135707 & 0.215584 & -0.015673 \\
1 & -1.631219 & 1.525725 & 0.351479 \\
7 & 0.949763 & 1.025175 & 0.016908 \\
1 & 1.034156 & 2.031261 & -0.022373 \\
7 & 0.290600 & -1.040419 & -0.027298 \\
7 & 1.653287 & -0.971835 & -0.012965 \\
7 & 2.073047 & 0.241815 & 0.014235
\end{tabular}

\begin{tabular}{|c|c|c|}
\hline & & \\
\hline 0.086032 & 0.223898 & -0.113763 \\
\hline-0.881933 & 1.124667 & 0.099441 \\
\hline-2.012329 & 0.372436 & 0.155182 \\
\hline-1.711208 & -0.896038 & -0.006454 \\
\hline-0.366916 & -1.032156 & -0.182403 \\
\hline 1.437044 & 0.592460 & -0.286728 \\
\hline 2.429044 & -0.358223 & 0.199121 \\
\hline 1.571738 & 1.522864 & 0.097077 \\
\hline 3.433328 & 0.037165 & -0.002392 \\
\hline 2.310273 & -1.302417 & -0.339187 \\
\hline 2.341605 & -0.581252 & 1.279088 \\
\hline 5 & & \\
\hline 2.405532 & 0.318445 & 0.000052 \\
\hline 1.405020 & -0.706698 & -0.000033 \\
\hline 3.360908 & -0.205644 & 0.000028 \\
\hline 2.328841 & 0.938155 & -0.901888 \\
\hline 2.328818 & 0.938034 & 0.902073 \\
\hline 0.130158 & -0.323837 & -0.000029 \\
\hline-0.341055 & 0.945860 & 0.000107 \\
\hline 0.124945 & 1.842078 & 0.000065 \\
\hline-0.892346 & -1.154883 & -0.000135 \\
\hline-1.706778 & 0.886980 & -0.000088 \\
\hline-2.002366 & -0.367484 & 0.000095 \\
\hline
\end{tabular}

Conjugate base of 5 


$\begin{array}{rrrr}6 & -0.097491 & -0.270190 & 0.000031 \\ 7 & 0.917562 & -1.133852 & 0.000080 \\ 7 & 2.008194 & -0.324024 & 0.000051 \\ 7 & 1.634524 & 0.938099 & -0.000158 \\ 7 & 0.274327 & 1.011225 & -0.000103 \\ 8 & -1.398526 & -0.701579 & 0.000094 \\ 6 & -2.365361 & 0.335084 & 0.000004 \\ 1 & -3.340853 & -0.163567 & 0.000066 \\ 1 & -2.268027 & 0.973443 & 0.888226 \\ 1 & -2.268050 & 0.973265 & -0.888349 \\ & & & \\ & & & \\ 7 & -2.290057 & 0.422843 & -0.523947 \\ 7 & -2.078426 & -0.832563 & -0.302125 \\ 7 & -0.863778 & -1.031553 & 0.281282 \\ 6 & -0.310566 & 0.162321 & 0.420160 \\ 7 & -1.186091 & 1.066567 & -0.079241 \\ 6 & 1.065222 & 0.471188 & 0.956607 \\ 7 & 2.151777 & 0.450007 & -0.020085 \\ 6 & 2.426440 & -0.856717 & -0.623569 \\ 1 & 1.576236 & -1.300353 & -1.164044 \\ 1 & 3.268700 & -0.749425 & -1.314287 \\ 1 & 2.724801 & -1.555541 & 0.165999 \\ 1 & 2.003965 & 1.153292 & -0.740643 \\ 1 & 1.289206 & -0.268490 & 1.732058 \\ 1 & 1.054935 & 1.456563 & 1.438467 \\ 1 & -1.138400 & 2.076103 & -0.127925\end{array}$

Conjugate base of 6

$\begin{array}{rrc}-2.098975 & -0.860762 & 0.073740 \\ -2.221829 & 0.336830 & 0.621356 \\ -1.116246 & 1.068843 & 0.362804 \\ -0.339534 & 0.252973 & -0.367239 \\ -0.912643 & -0.945501 & -0.565202 \\ 1.024143 & 0.607101 & -0.891962 \\ 2.128488 & -0.261955 & -0.425817 \\ 2.340412 & -0.177635 & 1.021014 \\ 1.436967 & -0.377475 & 1.620855 \\ 3.122394 & -0.890918 & 1.312135 \\ 2.694497 & 0.830658 & 1.273340 \\ 1.852839 & -1.218681 & -0.648552 \\ 1.259381 & 1.640214 & -0.607343 \\ 1.032225 & 0.559382 & -1.989488\end{array}$

